\title{
A Comparison between an Advanced High-Strength Steel and a High-Strength Steel Due to the Spring back Effect
}

\author{
Erika Aparecida da Silva ${ }^{1}$, Luiz Fernando Vargas Malerba Fernandes ${ }^{3}$, \\ José Wilson de Jesus Silva, ${ }^{3,5}$, Rosinei Batista Ribeiro, ${ }^{3,4}$, \\ Marcelo dos Santos Pereira ${ }^{1}$, JoëlAlexis ${ }^{2}$ \\ ${ }^{1}$ Universidade Estadual Paulista - UNESP, Campus de Guaratinguetá, SP, Brazil \\ ${ }^{2}$ Université de Toulouse, LaboratoireGénie de Production, ENIT/INPT, Toulouse, France \\ ${ }^{3}$ Centro Universitário Teresa D'Ávila - UNIFATEA - Rede Salesianas, Lorena, SP, Brazil \\ ${ }^{4}$ Faculdade de Tecnologia do Estado de São Paulo - FATEC, Cruzeiro, SP, Brazil \\ Associação Educacional Dom Bosco - AEDB, Resende, RJ, Brazil
}

\begin{abstract}
In this project, it is planned to carry out a complete microstructural characterization of advanced high strength steels (AHSS) currently used in the automotive industry. These steels have an elastic effect, called spring back effect, during the sheet metal forming of parts. This spring back is the main problem of this family of steels during the production process of the structural components of the automotive industry: especially for dimensional problems in automotive structural components. It is therefore necessary to evaluate changes in microstructure during the stages of sheet metal forming in order to know precisely the microstructural and mechanical behavior of material after being subjected to this effect. Steels studied in this research work are currently used by all major automobile industries, which are dual-phase (DP) 600 and 780. The spring back effect mechanical characterization was performed by means of a mechanical bending test, called as Air Bending. The results indicate that the spring back effect in the DP 780 has the highest spring back rate due to its high mechanical strength due to Hall-Petchphenomenom. The comparison of analyses EBSD before and after bending tests showed a relation between the misorientation of grains and the spring back effect. The results obtained by microstructure analysis of EBSD confirmed the tendency of DP780 absorb a greater amount of energy during the deformation, thus resulting in greater springback effect.
\end{abstract}

Keywords: springback, dual-phase, steels, air bending, EBSD, industries.

\section{Introduction}

From the 1950s, there was a concern of the automotive industry to produce steel with high formability and low price, and this influenced in changes in vehicle models. In the 1970s, due to the oil crisis, many countries took serious measures to reduce and rationalize energy consumption. The automobile industry then had to find solutions, bringing development sinaerodynamics and reducing vehicles weight. And, in all countries, there can be no grow thin the automotive sector without giving due importance to the steel mills [1].

The automobile industry has taken steps such as reducing the size of vehicles, replacement of materials which are traditionally used for aluminum and plastic, and replacement of carbon steels. As a result, steelmakers aimedprimarily to promote the as sembly of advanced materials with high strength, ensuring an increased structural integrity, an increased resistance to shock, with a lower cost to the final product [2].

A system sets the conventional high-strength steel (HSS) as those having yield strength between 210 and $550 \mathrm{MPa}$ and tensile strength between 270 and $700 \mathrm{MPa}$, while the high strength advanced steel (AHSS) has a yield strength that is greater than $550 \mathrm{MPa}$, and tensile strength greater than $700 \mathrm{MPa}$ [3].

The main difference between the HSS and the AHSS is their microstructure. HSS steels are ferriticpearlitic of a single phase. AHSS are steels, which mainly contain a microstructure with a different phase than ferrite, for example, martensite, bainite, austenite and / orretaine daustenite in sufficient quantities to produce their own mechanical properties.

However, the widespread use of AHSS in the automotive industry is limited due to challenges in formability, metal sheet, tool life, and to the springback effect. It is a major problem that compromises the mass production of automotive structural components with AHSS [4,5].

According to Ramezani [6], it is a partially undesirable change occurring in steel sheets as a result of the constraints removal after their sheet metal forming process.

The two types of steels studied in this work are among the major steels used by automobile industries nowadays because they are high strength steels, being dual-phase steel: DP600 and DP780, which are AHSS and this numbering corresponds to their respective limits of resistance in MPa.The dual phase steels consist of a ferritic structure array containing a second hard phase of martensite in the form of Islands. This type of steel is 
one of the AHSS to better balance between strength and ductility. Due to the high hard ening coefficient, the dual phase steel ensures greater formability than conventional steels, which have the same resistance range [7].

2.1Tensile tests

\section{Experimental Procedure}

Tensile tests were performed on universal testing machine Instron according to ASTM E 8M.

\subsection{Metallography before the sheet metal forming}

The following metallographic preparation processes are standardized by ASTM ID: E 3-10 (2007). After sectioning in the longitudinal direction of rolling, the specimens were subjected to hot embedding with bakelite. During the grinding, the following abrasives were used: 220, 320, 400, 600, 1000, and 1200 mesh. The polishing was performed in an OP-U solution and distilled water, subjecting the specimens to a rotation of 600 rpm. The chemical etching was done with a $2 \%$ Nital solution to reveal the grain bound aries offerrite and constituents [8,9].The micrographs were obtained using the NIKON optical microscope, model EPIPHOT 200.

\subsection{Sheet metal forming tests: Air Bending}

Sheet metal forming tests were run to two different sizes of steel blanks:

1. Test specimens were made from the same material as delivered and sectioned at the following dimensions: $80 \mathrm{~mm}$ long, $30 \mathrm{~mm}$ wide and $1.50 \mathrm{~mm}$ thick for DP600 and $1.27 \mathrm{~mm}$ thick for DP780. Such dimensions of the specimens were made according to the parameters defined for the unconstrained cylindrical bending test presented at the Numisheet conference 2002 [10].

2. Test specimens were made from the same material as delivered and sectioned at the following dimensions: $140 \mathrm{~mm}$ long, $40 \mathrm{~mm}$ wide and $1.50 \mathrm{~mm}$ thick for DP600 and $1.27 \mathrm{~mm}$ thick for DP780. Such dimensions of the specimens were made according to the parameters defined by some authors [11, 12].

The specimens were subjected to a test called the three-point air bending, in which the specimen is subjected to a punch with the cylindrical body. This experiment was made in according to the ASTM ID: E 29009 standards.

The punch had a $5 \mathrm{~mm}$ radius and the distance between the supports of the die was $13 \mathrm{~mm}$, according to the ASTM to a sample thickness of about $1 \mathrm{~mm}$. The three-point air bending was performed in a universal Shimadzu testing machine, Autograph AG-X, model $50 \mathrm{kN}$.

The specimens were subjected to sheet metal forming until the internal angle of bending reached a predetermined value. The value selected for the internal angle bending was 30 degrees for each bend, using two replicates in the same material. The punch was removed from the material 20 seconds after reaching the initial bending angle $\left(30^{\circ}\right)$, and then, the new bend angle measurement was made to determine whether there was a spring back effect or not. For this measurement, it was used the Image J 1.45 software for processing images photographed on an Olympus digital camera. Such measurements continue to be made for a period of $12 \mathrm{~h}, 24 \mathrm{~h}$, $48 \mathrm{~h}, 72$ hand $96 \mathrm{~h}$ after sheet metal forming. Completed the $96 \mathrm{~h}$ after the mechanical bending, the resulting bending angle was subtracted from the initial angle of bending, which was of $30^{\circ}$, and this subtraction resulted in a total springback angle $(\theta 1+\theta 2)$, as shown in Figure 1 .

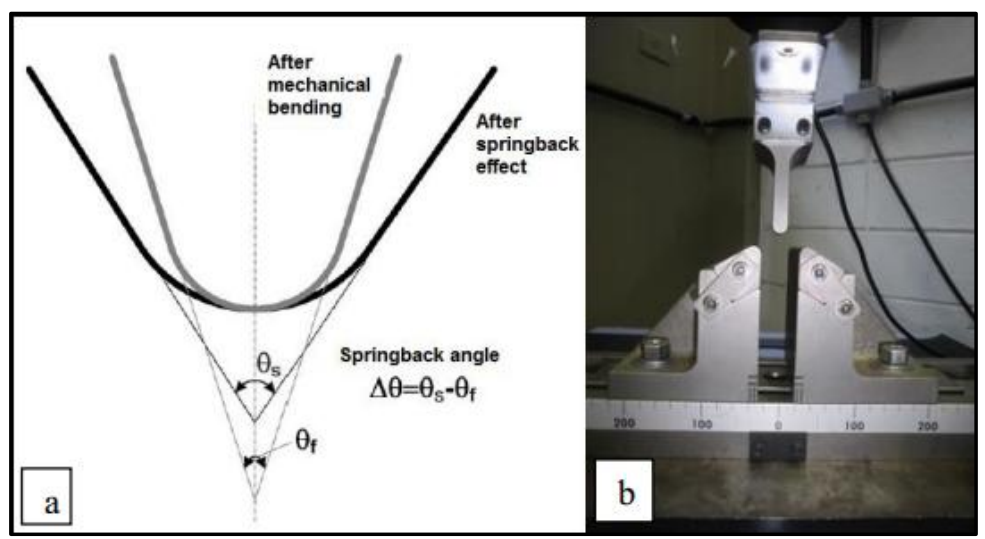

Figure 1: (a): Definition of springback angle [12]. (b): Device mounted universal testing machine for testing three-point bending in air or Air bending test.

\subsection{Metallography after the Sheet Metal Forming}

Test specimens were made from materials that were subjected to a mechanical bending test after 96hours of evaluation. The region of the steel plates chosen to obtain the specimens was the one where a curvature was formed due to bending. The specimens were cut in a longitudinal direction, i.e., in the direction of the steel plates lamination by dividing them in the middle. This material was embedded in order to expose its 
inner surface in order to obtain samples of the region that was deformed during the bending. The metallographic followed in the same manner as in the pre sheet metal forming test, with the use of hot embedding and polishing, and the chemical etching was made with a $2 \%$ Nital solution.

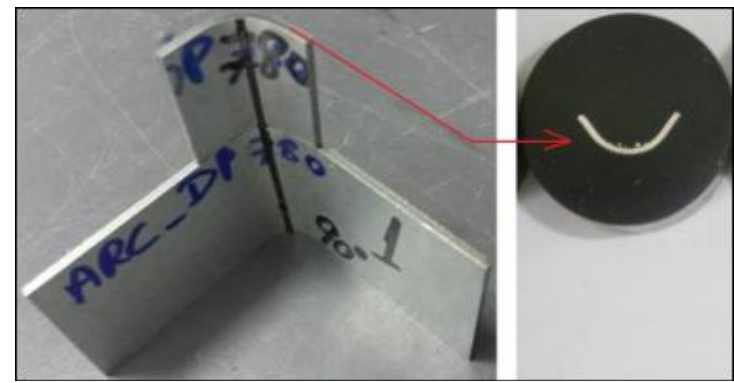

Figure 2: Deformed region after the sheet metal forming test, which was submitted to metallographic characterization

\subsection{Electron backscatter diffraction (EBSD)}

The EBSD is a technique that consists in placing a sample with perfectly flat surface inclined to 70 degrees with the electron beam incident. Back scattered electrons generate a diffraction pattern, which appears in the form of bands (Kikuchi bands), which can be viewed on a video monitor along with the image the place of incidence of the beam.

The samples were subjected to electrolytic polishing before being placed in the inside of SEM.

The EBSD technique was used in this study to determine the size of the grains and to determine the type of structure of grains (recrystallized, deformed and under structured).The equipment used was the JEOLJSM -7000F, software Channel 5 from Oxford Instruments HKL.

\subsection{Tensile tests}

\section{Results and Discussion}

The results of the tensile tests showed that the steel traction DP 780 has a greater mechanical resistance compared to steel DP 600, as expected. It was also possible to verify that the DP 780 presents a superior modulus of resilience $\frac{\sigma^{2} y i e l d}{2 * E}$ to theDP 600 and consequently, the DP 780 steel features a smaller percentage of elongation.

Table 1: Results of tensile tests for the steels dual phase 600 and 780

\begin{tabular}{|l|l|l|l|l|l|}
\hline Steels & $\begin{array}{l}\text { Yield Strength } \\
\sigma(\mathbf{M P a})\end{array}$ & $\begin{array}{l}\text { Tensile strength } \\
\sigma \mathrm{e}(\mathbf{M P a})\end{array}$ & $\begin{array}{l}\text { Young's Modulus } \\
\mathbf{E}=\Delta \sigma \mathrm{e} / \Delta \varepsilon\end{array}$ & $\begin{array}{l}\text { Modulus of Resiliense } \\
\mathbf{U r}=\sigma \mathbf{e}^{2} / \mathbf{2 E}(\mathbf{K P a})\end{array}$ & Elongation \\
\hline DP 600 & $669.66 \pm 0.079$ & $421.02 \pm 8.41$ & $200.47 \pm 13.62$ & $442.79 \pm 13.02$ & $31.95 \pm 1.92$ \\
\hline DP 780 & $864.43 \pm 31.55$ & $604.90 \pm 1.86$ & $204.45 \pm 1.02$ & $894.84 \pm 1.02$ & $23.73 \pm 3.26$ \\
\hline
\end{tabular}

\subsection{Metallography before the sheet metal forming}

According to Figure 3, note that the size of the grains of the DP 780 are visibly smaller than grains of DP 600 steel.

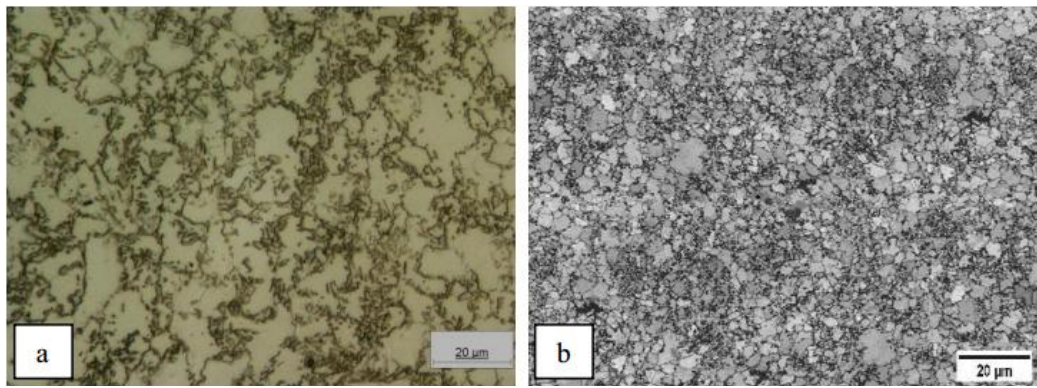

Figure 3: Optical microscopy before the sheet metal forming. Reagent: Nital 2\%. (a): DP600. (b): DP 780. $500 \mathrm{X}$

\subsection{Air Bending tests}

The spring back effect was greater for steel DP780, as expected for a higher mechanical strength steel.

The spring back was also greater when the steel sheet was smaller, as noted by Figure 4. 


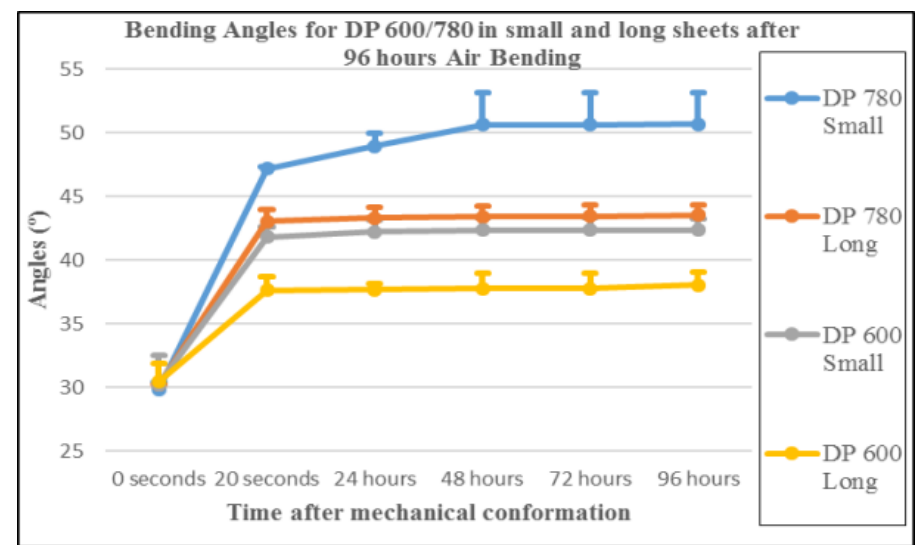

Figure 4: Bending angles after air bending, where, DP 600 and DP 780 Small have dimensions: 80 x 30mm; and DP 600 and DP 780 Long have dimensions: 140 x $40 \mathrm{~mm}$.

When the size of the specimens were changed, It caused a consider able change in the spring back effect. Using smaller specimens, the difference between the springback of DP 780 and 600 was 8.735. However, when increased the dimensions of specimens, this difference has decreased to 5.765 .

Table 2: Bending angles after air bending tests, total springback after 96 hours and difference between spring back angles for the DP 600 and DP 780 in different dimensions of sheets.

\begin{tabular}{|l|l|l|l|l|l|}
\hline Typeofthespecim & Steels & Value for bending angle for each time after air bending test & Total Springback & Differencespringback \\
\cline { 3 - 6 } & & 0 seconds & 96 hours & $(96$ hours -0 seconds $)$ & Between DP 600 and 780 \\
\hline $\begin{array}{l}\text { SmallSheet } \\
(80 \times 30)\end{array}$ & DP 600 & $30.204 \pm 2.328$ & $42.329 \pm 0.908$ & 12.125 & 8.735 \\
\cline { 2 - 6 } & DP 780 & $29.794 \pm 0.589$ & $50.654 \pm 2.463$ & 20.861 & \\
\hline $\begin{array}{l}\text { LongSheet } \\
(140 \times 40) \mathrm{mm}\end{array}$ & DP 600 & $30.427 \pm 1.440$ & $38.026 \pm 1.035$ & 7.599 & \\
\cline { 2 - 6 } & DP 780 & $30.144 \pm 0.063$ & $43.508 \pm 0.835$ & 13.364 & \\
\hline
\end{tabular}

\subsection{Metallography after the sheet metal forming}

In Figures5 and 6, note that the grains are visibly aligned in the same direction after mechanical sheet metal forming.
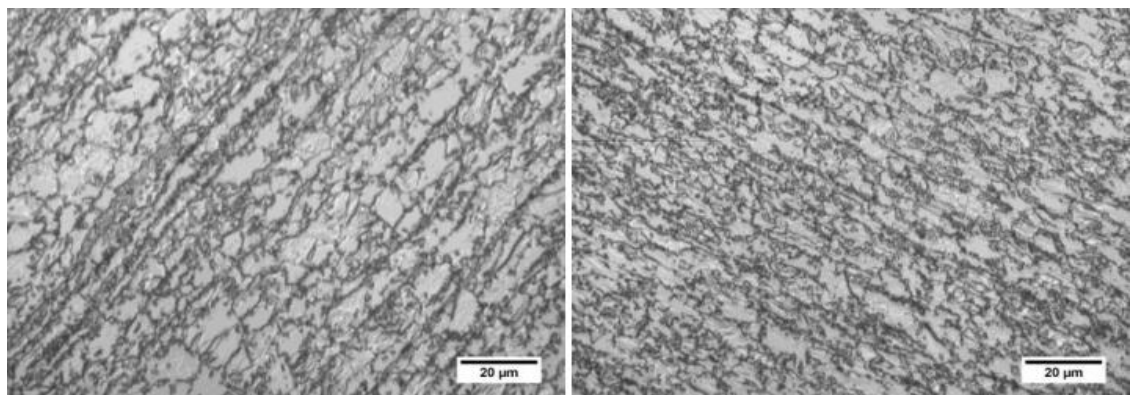

Figure 5: Optical microscopy after air bending test to 30 degrees for the DP 600. Reagent: Nital 2\%. 500X.
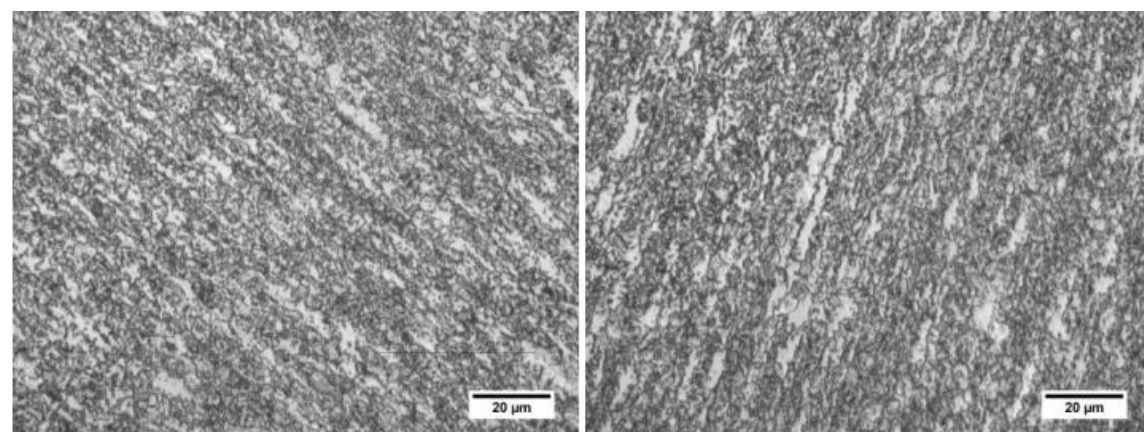

Figure 6: Optical microscopy after air bending test to 30 degrees for the DP 780. Reagent: Nital 2\%. 500X. 


\subsection{EBSD}

Analyzing the upper surface of the steel, note that grain size of DP 600 is larger than the size of the grains of the DP 780.Comparing the Table 2 and 3, it is observed that the DP 780 had the highest springback rate due to its high mechanical strength due to Hall-Petchphenomenom $[13,14]$.

$$
\sigma_{y}=\sigma_{0}+\frac{K_{y}}{\sqrt{d}}
$$

Figure 7: Hall-Petch equation, where $\sigma_{\mathrm{y}}$ is the yield stress, $\sigma_{\mathrm{o}}$ is a materials constant for the starting stress for dislocation movement (or the resistance of the lattice to dislocation motion), $\mathrm{k}_{\mathrm{y}}$ is the strengthening coefficient

(a constant specific to each material), and $\mathrm{d}$ is the average grain diameter

Table 3: Results from EBSD for steels DP 600 and DP 780 with respect to the size of the grains. Analysis before air bending tests in two surfaces of the sheets.

\begin{tabular}{|l|l|l|}
\hline \multicolumn{2}{|c|}{ Before Air Bending } \\
\hline Region of the sheet & Steel & Grains' diameter $(\boldsymbol{\mu m})$ \\
\hline \multirow{2}{*}{ From upper surface } & DP 600 & $6.113 \pm 4.389$ \\
\cline { 2 - 3 } & DP 780 & $1.831 \pm 1.946$ \\
\hline \multirow{2}{*}{ From thickness surface } & DP 600 & $1.004 \pm 0.957$ \\
\cline { 2 - 3 } & DP 780 & $1.223 \pm 0.831$ \\
\hline
\end{tabular}

According to table 4, note that the diameter of grains increased after air bending test, while the mean misorientation angle decreased. Misorientation is the difference in crystallographic orientation between two crystalline structures and this difference decreased after sheet metal forming, in the steel DP780 (Figure8).This confirms ob served in Figure 6, when it was noticed that grains were visibly aligned in the same direction after sheet metal forming.

Table 4: Results from EBSD for steel DP 780 in only thickness surface, before and after sheet metal forming.

\begin{tabular}{|l|l|l|l|}
\hline DP 780 & \\
\hline From thickness surface & \\
\hline Steel metal forming & Grains diameter $(\boldsymbol{\mu m})$ & Aspectratio & Mean Misorientation angle $\left({ }^{\mathbf{0}}\right)$ \\
\hline Before air bending & $1.223 \pm 0.831$ & $1.885 \pm 0.702$ & $1.018 \pm 0.679$ \\
\hline After air bending & $1.666 \pm 1.402$ & $1.778 \pm 0.612$ & $0.947 \pm 0.652$ \\
\hline
\end{tabular}
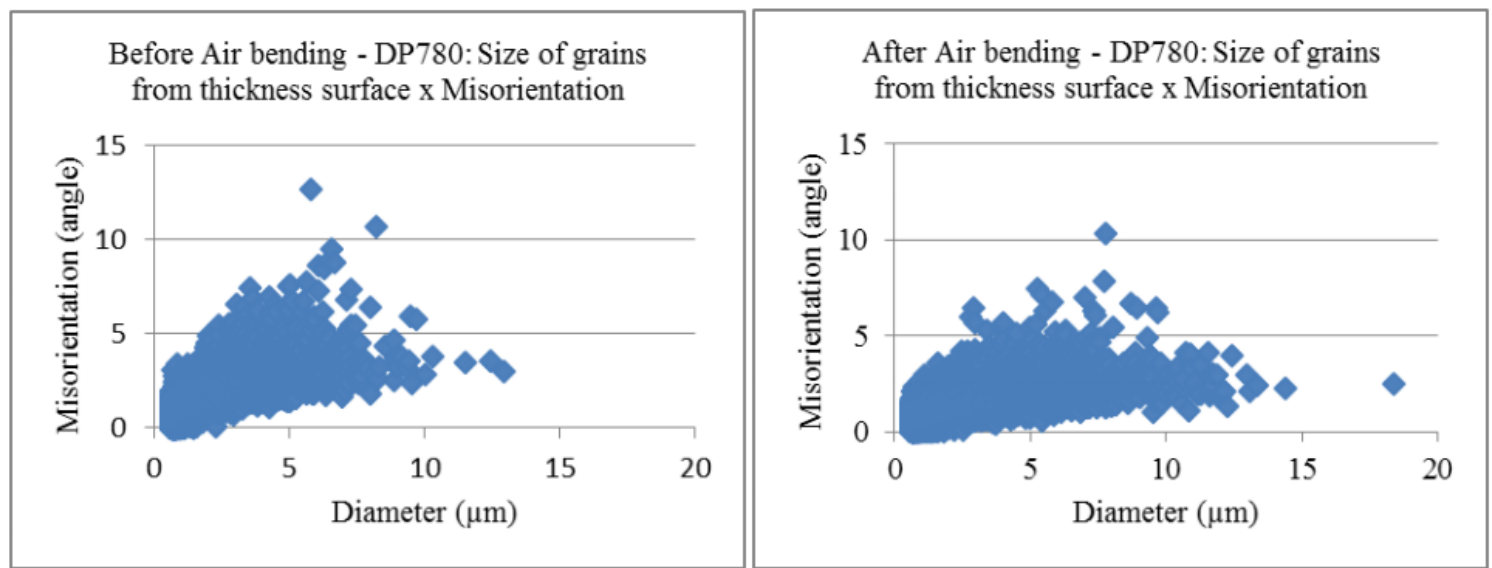

Figure 8: Dispersion relating the size of the grains with the misorientation angle for the steel DP 780, before and after sheet metal forming.

In Figure 9, it is interesting to note that with respect to the orientation of crystallographic plans, in steel DP 780, there have been some changes. Prior to sheet metal forming: in (a): in the area of the top surface, there was a predominance of blue color, indicating the orientation (111) and (b): in the area of the surface of the thick, there was a predominance of green color, indicating the orientation (101). After sheet metal forming, in the region of surface thickness, colors were balanced, indicating a balance among the three crystallographic orientations. 


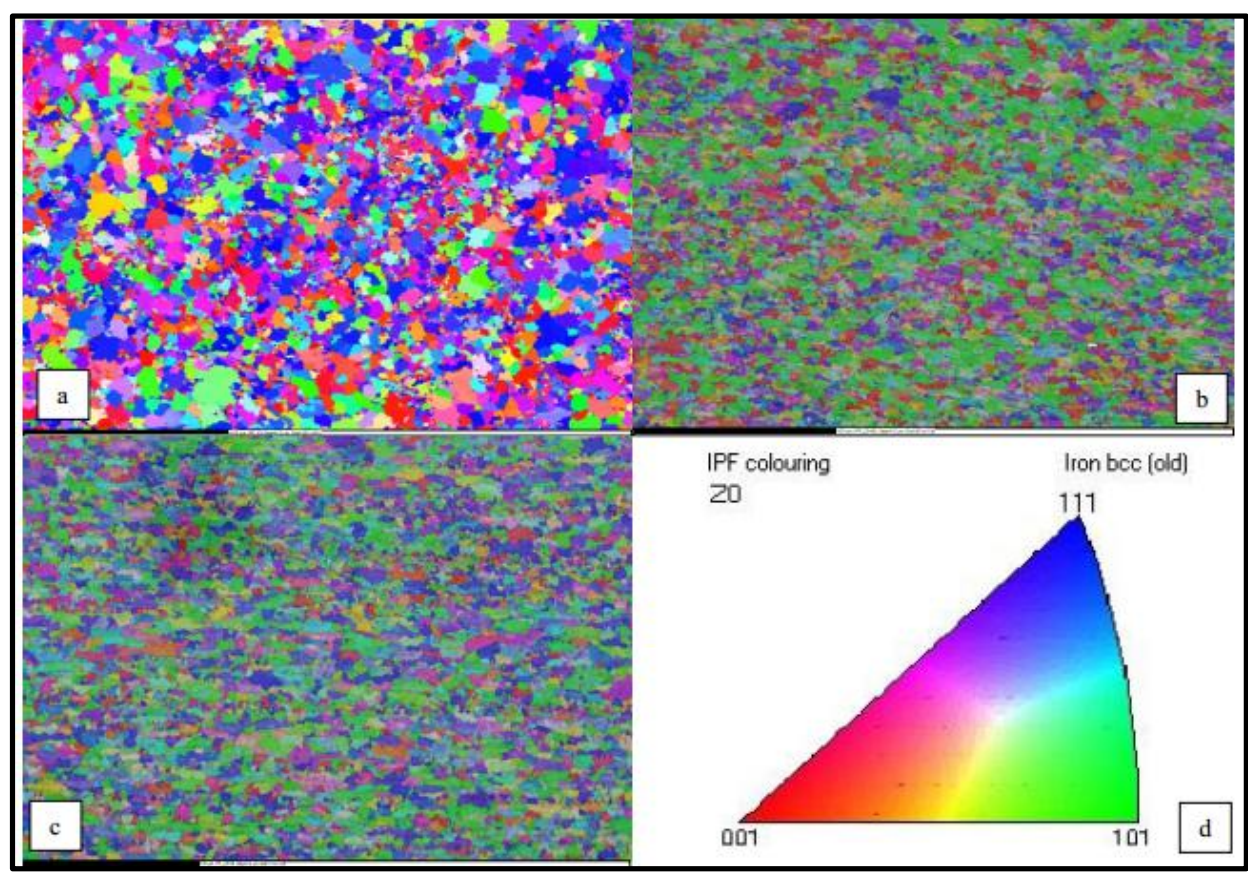

Figure 9:Crystallographic orientation plans for the DP 780. (a): Upper surface before air bending. (b): Thickness surface before air bending. (c): Thickness surface after air bending up to 30 degrees. (d): Legend indicating the Miller indices: crystallographic orientations.

Before the air bending test, the percentage of recrystallized grains of steel DP 600 was higher than in DP 780, both in the area of upper surface as the thickness surface of the sheet. Before the air bending, DP 780 showed a higher rate of grain under structured, in the area of upper surface and a higher rate of deformed grainslocated in the region of thickness surface (Table 5).

With respect to the DP 780, the rate of grains under structured located in the surface region of the thickness, which was lower than the rate of the DP 600, became higher than the DP 600 after the air bending.

Table 5: Percentage of grains recrystallized, under structured and deformed for DP 600 and 780, before and after sheet metal forming, along two surfaces.

\begin{tabular}{|l|l|l|l|l|l|l|}
\hline \multicolumn{8}{|c|}{ Type of the grains' structure } \\
\hline Conformation & Region of the sheet & Steel & Recrystallized & UnderStructured & Deformed \\
\hline \multirow{3}{*}{ Before air bending } & & DP 600 & 67.3 & 28.8 & 3.9 \\
\cline { 3 - 6 } & & DP 780 & 33.9 & 58.8 & 7.3 \\
\cline { 2 - 6 } & \multirow{2}{*}{ From thickness surface } & DP 600 & 7.85 & 43.9 & 48.3 \\
\cline { 2 - 6 } & & DP 780 & 2.19 & 17.3 & 80.5 \\
\hline After air bending & From thickness surface & DP 780 & 4 & 70.5 & 25.5 \\
\hline
\end{tabular}

\section{Conclusions}

It is concluded that, with respect to springback effect, this is higher according to: higher rate of resiliency module, smaller dimensions of specimens, smaller diameter of grains which gives a greater mechanical resistance according to the Hall-Petch equation.

The results from the EBSD technique showed that the degree of misorientation decreases after the steel suffering mechanical bending, so that the grains are trying to align in the same direction, decreasing the difference between the possible crystallographic orientations. This was confirmed when looking at the maps generated from the Miller indices, which showed that after bending, there was a balance in the colors indicative of crystallographic orientations. Finally, in the case of steel DP 780, the grains of the thickness surface, that were deformed before the air bending, became under structured after the air bending.

\section{Acknowledgements}

The authors are grateful to the Laboratoire Genie de Production - ENIT,INP Toulouse, France, also thank the company Arcelor Mittal Brasil by donations of steel samples to UNESP and CAPES for financial support. 


\section{References}

[1] Nogueira, A.L., Silva, M.J. Da carroça ao ultraleve reduction. Metalurgia \& Materials, vol. 57, n.511, august, 2001.

[2] Kishida, K. (2000). High strength steel sheets for light weight vehicle. Nippon steel technical report, 12-16.

[3] Keeler, S., \& Kimchi, M. (2015). Advanced High-Strength Steels Application Guidelines V5.World Auto Steel.

[4] Placidi, F., Vadori, R., Cimolin, F., \& Campana, F. (2008). An efficient approach to springback compensation for ultra high strength steel structural components for the automotive field.

[5] Yu, H. Y. (2009). Variation of elastic modulus during plastic deformation and its influence on springback. Materials \& Design, 30(3), 846-850.

[6] Ramezani, M., Ripin, Z. M., \& Ahmad, R. (2010). Modelling of kinetic friction in V-bending of ultra-high-strength steel sheets. The International Journal of Advanced Manufacturing Technology, 46(1-4), 101-110.

[7] Barnes, S. J., Bhatti, A. R., Steuwer, A., Johnson, R., Altenkirch, J., \& Withers, P. J. (2012). Friction stir welding in HSLA-65 steel: part I. Influence of weld speed and tool material on microstructural development. Metallurgical and Materials Transactions A, 43(7), 2342-2355.

[8] Vander Voort, G. F. Metallography, Principles and Practice. New York: McGrawHill, 1999. 752p.

[9] Dias, E. X. (2014). Caracterização metalográfica de um aço de fases complexas (CP).

[10] Yang, D. Y., Oh, S. I., Huh, H., \& Kim, Y. H. (2002, October). NUMISHEET 2002. In Proceedings of the 5th International Conference and Workshop on Numerical Simulation of 3D Shell Forming Processes-verification of Simulation with Experiment.

[11] Ján, S., \&Miroslav, J. (2012). Springback prediction in sheet metal forming processes. Journal for Technology of Plasticity, 37(1).

[12] Lee, M. G., Kim, S. J., Wagoner, R. H., Chung, K., \& Kim, H. Y. (2009). Constitutive modeling for anisotropic/asymmetric hardening behavior of magnesium alloy sheets: Application to sheet springback. International Journal of Plasticity, 25(1), 70-104.

[13] Hansen, N. (2004). Hall-Petch relation and boundary strengthening. Scripta Materialia, 51(8), 801-806.

[14] Kamikawa, N., Huang, X., Tsuji, N., \& Hansen, N. (2009). Strengthening mechanisms in nanostructured high-purity aluminium deformed to high strain and annealed. Acta Materialia, 57(14), 4198-4208. 